\title{
Magnetic Force Microscopy Analysis of Double-layered Perpendicular Magnetic Recording Media recorded by using Ring-type head. (Department of Applied Chemistry, Waseda University.)
}

Table I Magnetic properties of single-layered perpendicular magnetic recording medium and double-layered perpendicular magnetic recording media.

\begin{tabular}{l|c|c|c}
\hline Medium & A & B & C \\
\hline Upper layer & CoNiReP & CoNiReP & CoNiReP \\
\hline Thickness / um & 0.2 & 0.2 & 0.2 \\
Ms / emu cm & 350 & 350 & 350 \\
Hc - ) $/$ Oe & 1200 & 1200 & 1200 \\
\hline Underlayer & - & CoB & CoNiP \\
\hline Thickness / um $_{\text {Ms / emu cm }}^{-3}$ & & 0.03 & 0.03 \\
Hc(u)/Oe & & 1000 & 800 \\
\hline
\end{tabular}

\section{Medium A}
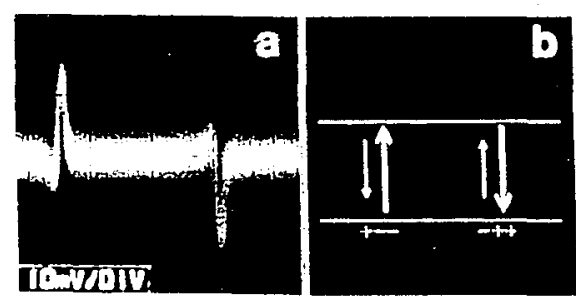

Table II. Read/Write conditions and Head parameters

\begin{tabular}{l|c} 
parameters. & \\
\hline Head & Mn-Zn ferrite \\
\hline Bs $/ \mathrm{kG}$ & 5 \\
\hline Gap length / $\mu \mathrm{m}$ & 0.37 \\
\hline Track width $/ \mu \mathrm{m}$ & 60 \\
\hline Coil turns & 15 \\
\hline Relative velocity $\mathrm{m} \cdot \mathrm{s}^{-1}$ & 1.0 \\
\hline
\end{tabular}

Medium B
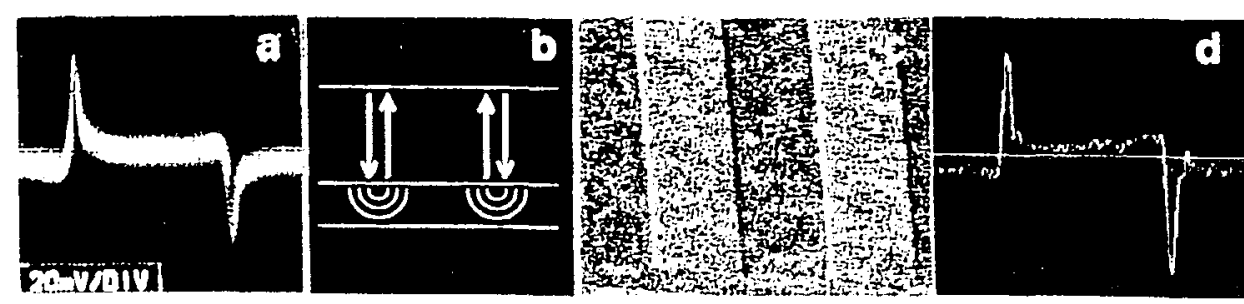

Medium C
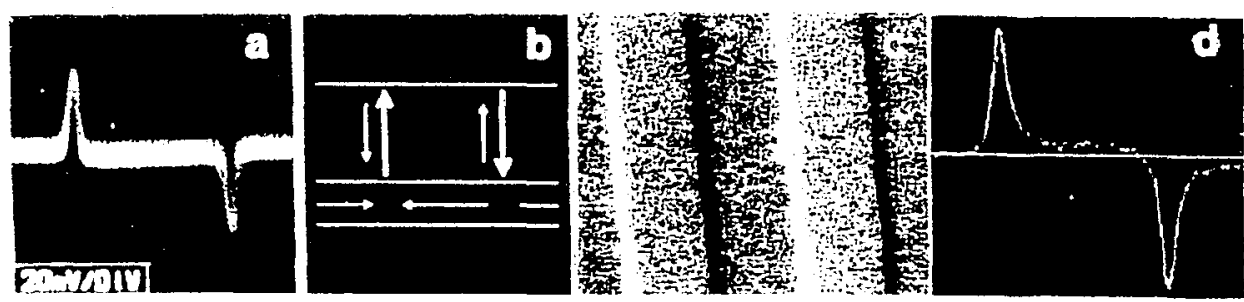

Fig. I Representative reproduced waveforms of the isolated pulse (a), presumed models for the recorded magnetization mode (cross-sectional view of the medium), showing the direction of residual magnetization vectors (b), representative top-view MFM images(c), and its averaged cross-sectional view (obtained from trimmed area) (d) of the media $A$. B, and $C$. The recording density is $2 \mathrm{kFRPI}$, the peak to valley distance in the waveform and MFM image is $12.74 \mathrm{~m}$. The full scale of (d) is \pm I. id (deg) 

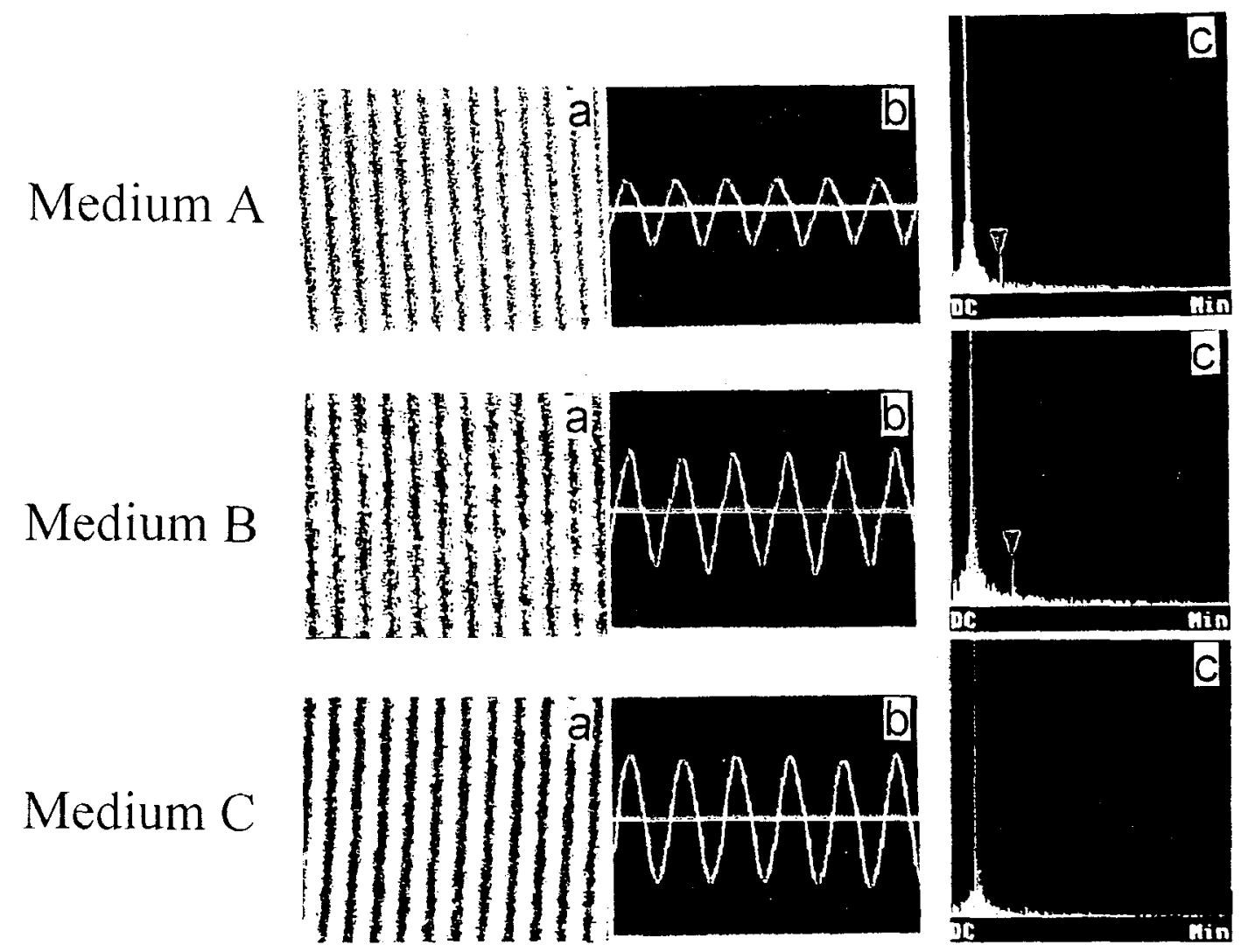

Fig. 2 Representative top-view MFM image (a), its averaged cross-sectional view (b), and the power spectrum (c) of the media $A, B$, and C.

(Supplementary data)

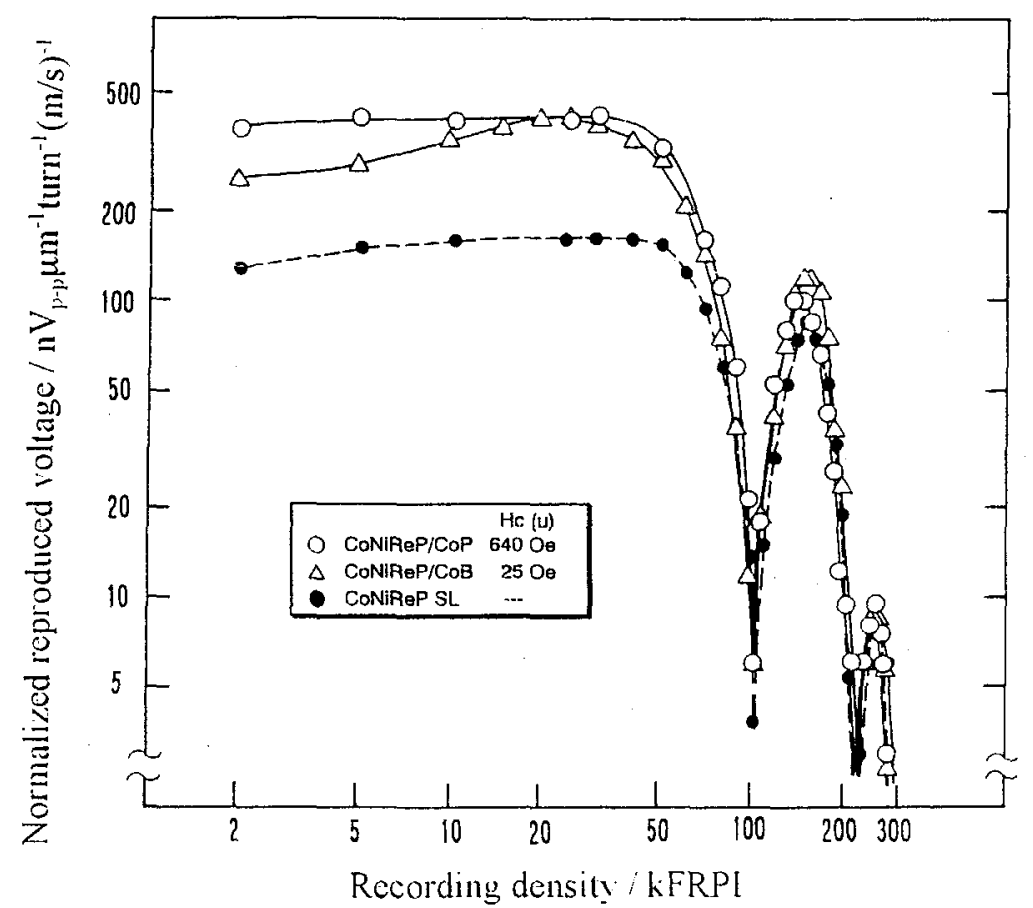

Fig. 3 Dependence of normalized reproduced voltage on recording density.

References

T. Homma, Y. Kurokawa, T. Nakamura, and T. Osaka : J. Fac. Sci. Techmol., B 14(2), 1184 (1996)

Y. Kurokawa and T.Osaka: J. Mag. Soc.Jpn., 19(S2), 131(1995) 